\title{
Wideband Array Processing Using a Two-Sided Correlation Transformation
}

\author{
Shahrokh Valaee and Peter Kabal, Member, IEEE
}

\begin{abstract}
A new method for broadband array processing is proposed. The method is based on unitary transformation of the signal subspaces. We apply a two-sided transformation on the correlation matrices of the array. It is shown that the twosided correlation transformation (TCT) has a smaller subspace fitting error than the coherent signal-subspace method (CSM). It is also shown that unlike CSM, the TCT algorithm can generate unbiased estimates of the directions-of-arrival, regardless of the bandwidth of the signals. The capability of the TCT and CSM methods for resolving two closely spaced sources is compared. The resolution threshold for the new technique is much smaller than that for CSM.
\end{abstract}

\section{INTRODUCTION}

A RRAY processing is a powerful tool for detecting and locating the signals arriving at a set of sensors. The sensors are distributed in space, and the signals received at each sensor are delayed versions of the signals generated by the sources. If the noise is uncorrelated between sensors. the signal-to-noise ratio (SNR) can be increased by adding the appropriately weighted outputs of the sensors. This is done by steering a bean toward the source direction (beamforming). Two sources can be resolved using a beamformer if their separation is larger than a beamwidth. To provide a better performance in the detection and localization of signals. a high-resolution method should be applied.

The objective of this paper is to introduce a new method for estimating the directions-of-arrival (DOA's) of wideband signals. Wideband processing arises in many applications such as audio conferencing, spread spectrum transmission. and passive sonar. A wideband signal is one that has a large bandwidth relative to its center frequency. A common approach to wideband array processing is based on sampling the signal spectrum at the output of the sensors. Each frequency bin creates a narrowband signal. In the so-called incoherent signal-subspace method (ISM), the narrow-band signals are processed as a vector to estimate the DOA's. Then, these results are combined to obtain the final solution [1]. Perfectly correlated (coherent) sources cannot be handled by this approach. Furthermore, the efficiency of this method deteriorates for closely separated sources and low SNR.

Manuscript received Juule 18,1992; revised March 28, 1994. This work w as supported by a Grant from the Natural Sciences and Engineering Research Council of Canada (NSERC). The associate editor coordinating the review of this paper and approving it for publication was Prof. Danicl Fuhrmann.

The authors are with the Department of Electrical Engineering. McGill University. Montreal, Canada H3A 2A7 and with INRS-Télécommunications,

Université du Québes, Verdun, Canada H3E 1 H6.

IEEE Log Number 9406918 .
The coherent signal-subspace method (CSM) [2] is an alternative to ISM that improves the efficiency of the estimation by condensing the energy of narrowband signals in a predefined subspace. This process is called focusing. A high-resolution method such as MUSIC [3] is then used to find the DOA's. The DOA's are estimated by determining the angular location of peaks in the spatial spectrum of the MUSIC algorithm. It has been shown [2] that CSM improves the resolution threshold and resolves coherent sources. Despite the fact that CSM is very effective in wideband signal detection and estimation, it suffers from an asymptotic bias of the peaks. The bias increases with the bandwidth of the sources and deviation of the focusing angles from the true DOA's. Recently, we have shown [4] that with a proper selection of the focusing frequency the estimation bias can be decreased. However. in general, an unbiased estimation of the DOA's is not possible using the CSM algorithm. We will show this in the present piper.

Two other techniques have evolved from the CSM method. The objective of these methods is to reduce the bias of the estimation. In [5], Buckley and Griffith propose a broadband signal-subspace spatial-spectrum estimation (BASS-ALE) algorithm. This method forms a broadband covariance matrix with the rank of the broadband signal representation subspace equal to one. The tradeoff is an increase in the computational complexity. In this method. the estimation bias is reduced by increasing the dimensionality of the location vectors. In [6], Krolik and Swingler propose an algorithm based on the steered covariance matrix (STCM). In their technique, delay elements are introduced at the front end of the array, and the covariance matrix is computed after the delays. With a proper choice of the delays, a steering bean can be formed. It has been shown that when the steering beam coincides with a true DOA, the STCM contains a dc term equal to the power of the corresponding source. Thus, by steering the space and locating the peaks of the dc component, the DOA's are estimated.

In this paper, we introduce a new technique for broadband array processing. Our method is similar to CSM in the sense that transformation of the signal subspaces is performed through focusing matrices. A high-resolution spectral estimation algorithm, such as MUSIC, is then applied to determine the DOA. In the new method, we apply a two-sided unitary transformation to the correlation matrix. In [7]. it has been shown that unitary transformations have good performance in terms of focusing loss and relative information index. The motivation for using the correlation matrices instead of the location matrices is based on the fact that most of the high- 
resolution spectral estimation algorithms use an eigenstructure decomposition of the correlation matrix. We show that the new method has a lower resolution threshold SNR and a smaller bias than CSM.

The paper is organized as follows. In the following section, we formulate the problem and review the CSM method. Section III presents some mathematical background for matrix approximation. In Section IV. we introduce the new method. Selection of the focusing matrix is based on minimizing the subspace fitting error. In Section V, we show that the TCT algorithm has a smaller subspace fitting error than CSM. In Section VI, the eigenvalues of the noise-free focused correlation matrix of the CSM and TCT algorithm are compared. It is shown that in the CS.M algorithm, the energy of signal is extended into the noise subspace. This signal extension acts as a colored noise with an unknown correlation matrix. In Section VII, using these results. we show that CSM cannot asymptotically generate unbiased estimates of the DOA's Section VIII contains a study on the performance of the proposed algoritlin under noisy conditions. There, we show that the generalized variance of the TCT algorithm is smaller than that for CSM. This results in a smaller variance of estimation. Section IX contains the simulation results.

\section{Coherent Signal Subspace Processing}

Consider an array of $p$ sensors exposed to $q<p$ far-field wideband sources. The signals of the sources can be partially or fully correlated. The output of the sensors is shown by $p$-vector $\boldsymbol{x}(t)$ with the $i$ th component

$$
n_{i}(t)=\sum_{i=1}^{q} s_{l}\left(t-\tau_{i}\left(\theta_{1}\right)\right)+n_{j}(t), \quad 1 \leq i \leq p
$$

where

s! lth source signal

$\theta_{l} \quad$ angle-of-arrival for the lth source

$\tau_{i}\left(\theta_{l}\right)$ propagation delay for the th source at the sensor with respect to the reference point of the array.

For a linear array with uniform spacing, $\tau_{i}\left(\theta_{l}\right)=(i-$ 1) $\frac{d}{c} \sin \theta_{l}$, where $d$ is the spacing between two consecutive sensors, and $c$ is the propagation velocity. It is also assumed that the observation is corrupted by an additive noise that is represented in the model by $n_{i}(1)$.

The array output in the frequency domain is represented by

$$
\boldsymbol{x}(\omega)=\boldsymbol{A}(\omega, \theta) \boldsymbol{s}(\omega)+n(\omega)
$$

where $\boldsymbol{s}(\omega)$ and $\boldsymbol{n}(\omega)$ are the Fourier transforms of the signal and the noise vectors, respectively. The $p \times q$ matrix $\boldsymbol{A}(\omega, \theta)=$ $\left[\boldsymbol{a}\left(\boldsymbol{w}, \theta_{1}\right) \cdots \boldsymbol{a}\left(\omega, \theta_{q}\right)\right]$ is called the location matrix of the array and is assumed to be full rank. In other words, the steering vectors $\boldsymbol{a}\left(\omega, \theta_{i}\right), i=1 \ldots \ldots$, are independent for every $\omega$.

The signal samples are generated independently by a complex Gaussian distribution with an unknown covariance matrix $\boldsymbol{S}(\omega)$. The noise samples are an i.i.d. sequence of complex Gaussian random vectors with unknown covariance matrix $\sigma^{2} \boldsymbol{I}$ and are independent of the signal samples. It is assumed that the noise is spatially white. This assumption can be relaxed if the correlation matrix of the noise is known but for a scale factor. In that case, a prewhitening step is required to create uncorrelated intersensor noise. From (2) and using the assumptions on the signal and noise samples, the covariance matrix of the observation vector at frequency $\omega$ is given by

$$
\boldsymbol{R}(\omega)=\boldsymbol{A}(\omega, \theta) \boldsymbol{S}(\omega) \boldsymbol{A}^{H}(\omega, \theta)+\sigma^{2} \boldsymbol{I}
$$

where the superscript $H$ represents the Hermitian transpose.

In practice, a sufficiently long duration of sensor output is observed. Then, the sampled data are divided into $N$ snapshots, each containing $J$ samples. In each snapshot, an FFT algorithm is used to transform the data into the frequency domain. Thus, $N$ sets of transformed data are available where each set contains $J$ frequency samples of the spectrum of the observation vector. We represent these samples by $\boldsymbol{x}_{j}, j=$ $1, \ldots, J$. It should be noted that each $x_{j}$ depends on the snapshot in which it has been produced. For simplicity of notation, this dependence has not been shown explicitly. In the sequel, we suppress the frequency variable representing $\boldsymbol{R}\left(\boldsymbol{\alpha}_{j}\right)$ by $\boldsymbol{R}_{j} \boldsymbol{S}\left(\boldsymbol{\omega}_{j}\right)$ by $\boldsymbol{S}_{j}, \boldsymbol{A}\left(\omega_{j}, \theta\right)$ by $\boldsymbol{A}_{j}$, and so on.

The signal subspace is defined as the column span of the steering matrix $A(\omega, \theta)$. The dimension of this subspace is determined by the number of sources. The orthogonal complement to this subspace is called the noise subspace. It is seen that the signal subspace is a function of the frequency $\omega$ and the angles-of-arrival $\theta$. Thus, the signal subspaces at different frequency bins are different.

The CSM algorithm [2] transforms these subspaces and overlaps them in a predefined subspace: the so-called focusing subspace. The focusing matrices $T_{j}$ 's are the solutions of the equation

$$
T_{j} \boldsymbol{A}_{j}=\boldsymbol{A}_{(1)}, j=1 \ldots J
$$

where $A_{0}$ is the focusing location matrix. The matrices $\boldsymbol{A}_{0}$ and $A_{j}$ are functions of the DOA's $\theta$. An ordinary beamforming preprocess gives an estimate of the angles-of-arrival that can be used in (4). Using the focusing matrices $T_{j}$, the observation vectors at different frequency, bins are transformed into the focusing subspace. In particular, new observation vectors are formed by

$$
\boldsymbol{y}_{j}=\boldsymbol{T}_{j} \boldsymbol{x}_{i} . \quad j=1, \ldots, J
$$

Then, these transformed observation vectors are used to construct the sample correlation matrices

$$
\boldsymbol{R}_{i}^{(y)}=\frac{1}{V} \sum_{l=1}^{N} \boldsymbol{y}_{i}^{(l)} \boldsymbol{y}_{i}^{(i)^{n}}
$$

where the transformed data vector for frequency bin $j$ and for batch $l$ is represented by $\boldsymbol{y}_{j}^{(t)}$. An average of these aligned correlation matrices over the frequency bins gives a universal focused sample correlation matrix that can be used for detection and estimation. If this matrix is represented by IR. we will have

$$
\boldsymbol{R}=\frac{1}{. J} \sum_{j=1}^{J} \boldsymbol{R}_{j}^{(y)}=\boldsymbol{A}_{0} \boldsymbol{R}_{s} \boldsymbol{A}_{0}^{H}+\sigma^{2} \boldsymbol{R}_{n}
$$


where

$$
\begin{aligned}
& R_{*}=\frac{1}{J} \sum_{j=1}^{J} S_{j}, \\
& R_{n}=\frac{1}{J} \sum_{j=1}^{J} T_{j} T_{j}^{H} .
\end{aligned}
$$

This transformation improves the efficiency of the estimation by condensing the energy of sub-bands in the focusing signal subspace. Yet, it creates a problem. It is seen that the focusing removes the whiteness of the noise. This in turn changes the SNR at the output of the processor. The focusing loss is defined as the ratio of the array SNR after and before focusing. Using this quantity, Hung and Kaveh [7] showed that the focusing is lossless if $T_{j}$ 's are unitary transformations. Specifically, they proposed using the transformation matrices obtained by the constrained minimization problem

$$
\begin{aligned}
& \underset{T}{\min } \| A_{0}-T_{j} A_{j} \mid \\
& \text { s.t. } T_{j}^{H} T_{j}=I
\end{aligned}
$$

for $j=1, \ldots, I$. They used the Frobenius matrix norm, which is defined by

$$
\|\boldsymbol{B}\|=\left[\sum_{i, j} b_{i, i}^{2}\right]^{\frac{1}{2}}=\left[\operatorname{tr}\left(\boldsymbol{B}^{H} B\right)\right]^{\frac{1}{2}}
$$

where $\operatorname{tr}(\cdot)$ stands for the trace of matrix.

\section{MATRIX APPROXIMATION}

The problem of approximating a given matrix by a matrix in a specified class arises in multivariate analysis, factor analysis, estimation of residuals in linear models, and the theory of generalized matrix inverse. In each case, a minimization problem is solved to obtain the closest distance between the two matrices. The distance between the matrices is measured with respect to an appropriate norm. One class of norms is known as the unirarily invariant norms. The Frobenius norm, used here, is one such norm which does not change when a unitary transformation is applied to the matrix.

We saw previously that the unitary transformation matrices of the CSM algorithm are the solutions of (10). In factor analysis, this is known as the problem of finding a procrustean transformation of $\boldsymbol{A}_{j}$. Assume that the singular value decomposition of $\boldsymbol{A}_{0} \boldsymbol{A}_{j}^{H}$ is represented by $\boldsymbol{V}_{j} \Sigma_{j} \boldsymbol{W}_{j}^{H}$. Then, the focusing matrix $\boldsymbol{T}_{j}$ that solves (10) is given by [8], [7]

$$
T_{j}=V_{j} W_{j}^{H} \text {. }
$$

In such a case, the error of transformation is

$$
\begin{aligned}
\sum_{j=1}^{J}\left\|\boldsymbol{A}_{0}-\boldsymbol{T}_{j} \boldsymbol{A}_{j}\right\|^{2}= & \sum_{j=1}^{J}\left[\left\|\boldsymbol{A}_{0}\right\|^{2}+\left\|\boldsymbol{A}_{j}\right\|^{2}\right. \\
& \left.-2 \Re \operatorname{tr}\left(\boldsymbol{A}_{0} \boldsymbol{A}_{j}^{H} \boldsymbol{T}_{j}^{H}\right)\right] \\
& =2 J p q-2 \sum_{j=1}^{J} \sum_{i=1}^{4} \sigma_{i}\left(\boldsymbol{A}_{0} \boldsymbol{A}_{j}^{H}\right)
\end{aligned}
$$

where $\sigma_{i}(\boldsymbol{B}), i=1, \ldots, q$ are the singular values of the matrix $B$ arranged in nonincreasing order, and $R$ stands for the real part of a complex number. In (13), we have used the equality

$$
\|\boldsymbol{A}\|^{2}=\sum_{i=1}^{q}\left\|\boldsymbol{a}_{i}\right\|^{2}=p q
$$

which holds for any arbitrary array manifold. We present a lemma that gives a lower bound to the error in (13).

Lemma 1: Let $\boldsymbol{A}: \boldsymbol{B} \in \boldsymbol{M}_{m \times n}$ (an $m \times n$ matrix) and $q=\min \{m, n\}$. Denote by $\sigma_{i}(\boldsymbol{A}), \sigma_{i}(\boldsymbol{B})$, and $\sigma_{i}\left(\boldsymbol{A} B^{H}\right), i=$ $1 \ldots q$ the nonzero singular values of the corresponding matrices arranged in nonincreasing order. Then

$$
\sum_{i=1}^{q} \sigma_{i}\left(\boldsymbol{A} B^{H}\right) \leq \sum_{i=1}^{q} \sigma_{i}(\boldsymbol{A}) \sigma_{i}(\boldsymbol{B}) .
$$

Proof: See Appendix A.

Lemma 1 is presented as a theorem in [9]. In Appendix A, we have provided a self-contained proof using a different approach.

From (13) and (15), it is seen that the error of transformation is lower bounded as

$$
\text { 2.Ipq } 2 \sum_{j=1}^{J} \sum_{i=1}^{q} \sigma_{i}\left(\boldsymbol{A}_{0}\right) \sigma_{i}\left(\boldsymbol{A}_{j}\right) \leq \sum_{j=1}^{J}\left\|\boldsymbol{A}_{0}-\boldsymbol{T}_{j} \boldsymbol{A}_{j}\right\|^{2} \text {. }
$$

This lower bound cannot be reached in general using the one-sided transformation of the CSM method.

Now, consider the alternative of a two-sided transformation. For such a transformation, we will be able to achieve the lower bound to the error. In a two-sided transformation, the objective is to find the unitary matrices $U$ and $V$ such that the following criterion is minimized:

$$
\begin{aligned}
& \min _{\mathrm{U} . \mathrm{V}}\left\|\boldsymbol{A}-\boldsymbol{U} B V^{H}\right\|^{2} \\
& \text { s.t. } \boldsymbol{U}^{H} \boldsymbol{U}=\boldsymbol{I} \text { and } \boldsymbol{V}^{H} \boldsymbol{V}=\boldsymbol{I} .
\end{aligned}
$$

Theorem 1: The solution of (17) is given by $U=\boldsymbol{E} X^{H}$ and $\boldsymbol{V}=F Y^{H}$, where $A=E \Sigma F^{H}$ and $B=X A Y^{H}$ are the singular value decompositions of $A$ and $B$, respectively, and the error of transformation is given by

$$
\mathcal{E}=\|\boldsymbol{A}\|^{2}+\|\boldsymbol{B}\|^{2}-2 \sum_{i=1}^{\varphi} \sigma_{i}(\boldsymbol{A}) \sigma_{i}(\boldsymbol{B})
$$

Proof: See Appendix B.

Corollary 1: If $\boldsymbol{A}$ and $\boldsymbol{B}$ are square Hermitian matrices, the transformation matrices $U$ and $V$ will be identical and equal to $E X^{H}$, where $E$ and $\boldsymbol{X}$ contain eigenvectors of $\boldsymbol{A}$ and $\boldsymbol{B}$, respectively.

From Theorem 1 and Lemma 1, it is seen that the error of transformation is minimized for the two-sided unitary transformation. Since the location matrix cannot be separately measured from the observation, the two-sided transformation of the location matrices is not practical. However, it is well known that for $q$ noncoherent sources, the space spanned by the location matrix is the same as the span of the eigenvectors of the correlation matrix that correspond to $q$ largest eigenvalues. Our method is based on two-sided transformation of 
the correlation matrix. This will be discussed in the following section.

\section{Two-Sided CORRElation TRANSFormation}

In this section, we introduce a new wide-band array processing technique based on transformation of the signal-subspaces into the focusing subspace. The transformation matrix at each frequency bin is unitary and minimizes the distance between the focusing subspace and the transformed signal subspace. In the new method, the transformation of the subspaces is performed through a two-sided transformation applied to the correlation matrix. The motivation for using the correlation matrix, instead of the location matrix, is attributed to two facts. First, a two-sided transformation can be applied, which results in a smaller error. This issue will be discussed in detail later. Second, many of the high-resolution methods for DOA estimation are based on the eigenstructure decomposition of the correlation matrix. Thus, the closer the transformed correlation matrices, the better the estimation results.

\section{A. The TCT Criterion}

Our method is based on transformation of the matrices

$$
\boldsymbol{P}_{j}=\boldsymbol{A}_{j} \boldsymbol{S}_{j} \boldsymbol{A}_{j}^{H}, \quad j=1, \ldots, J
$$

where $\boldsymbol{P}_{j}$ is the correlation matrix of the sensor output at the $j$ th frequency bin in a noise-free environment. Let $\boldsymbol{P}_{0}$ be the focusing noise-free correlation matrix. The TCT focusing matrices are found by minimizing

$$
\begin{aligned}
& \underset{\mathbf{U}}{\min }\left\|P_{0}-U_{j} P_{j} U_{j}^{H}\right\| \\
& \text { s.t. } U_{j}^{H} U_{j}=I
\end{aligned}
$$

for $j=1 \ldots, I$. From Corollary 1 , the solution of $(20)$ is obtained as

$$
\boldsymbol{U}_{j}=\boldsymbol{X}_{0} \boldsymbol{X}_{j}^{H}
$$

where $\boldsymbol{X}_{0}$ and $\boldsymbol{X}_{j}$ are the eigenvector matrices of $\boldsymbol{P}_{0}$ and $\boldsymbol{P}_{j}$, respectively. The matrix $\boldsymbol{U}_{j}$ can be used to transfer the observation vector $\boldsymbol{x}_{j}$ into $\boldsymbol{y}_{j}$ through

$$
\boldsymbol{y}_{j}=U_{j} \boldsymbol{x}_{j}
$$

The observation vectors $\boldsymbol{y}_{j}, j=1, \ldots, J$ are in the focusing subspace. Using (7)-(9), their correlation matrices can be averaged to find the universal focused sample correlation matrix.

In computing $U_{j}$, the matrices $\boldsymbol{A}_{j}$ and $\boldsymbol{S}_{j}$ are assumed to be known. In practice, a preprocessing step is required to estimate these matrices. A low-resolution beamformer is applied to estimate the number and the DOA's of the sources. Closely separated and correlated sources may not be resolved at this stage. Like [7], we add two extra focusing angles at $\pm 0.25 B_{W}$. (BeamWidth) of the estimated DOA. For instance, if the $i$ th DOA is found at $\hat{\theta}_{i}$ by the preprocessing, the focusing angles are chosen at $\left(\dot{\theta}_{i}-0.25 B_{W}, \hat{\theta}_{i}, \hat{\theta}_{i}+0.25 B_{\mathrm{W}}\right)$. In TCT, the number of focusing angles should be larger than the true number of sources. Using the results of this preprocessing step, an estimate of the location matrix $\boldsymbol{A}_{j}$ is obtained. Then, the eigenvalues of the sample correlation matrices $\hat{\boldsymbol{R}}_{j}, j=$ $1, \ldots, J$ are computed and sorted in decreasing order. The noise power at the $j$ th frequency bin is estimated by

$$
\hat{\sigma}_{j}^{2}=\frac{1}{p-q} \sum_{i=q+1}^{p} \lambda_{i}\left(\hat{\boldsymbol{R}}_{j}\right)
$$

where $\lambda_{i}(\boldsymbol{B})$ is the $i$ th eigenvalue of $\boldsymbol{B}$. The source correlation matrix is then found from

$$
\hat{\boldsymbol{S}}_{j}=\left(\boldsymbol{A}_{j}^{H} \boldsymbol{A}_{j}\right)^{-1} \boldsymbol{A}_{j}^{H}\left[\hat{\boldsymbol{R}}_{j}-\hat{\sigma}_{j}^{2} \boldsymbol{I}\right] \boldsymbol{A}_{j}\left(\boldsymbol{A}_{j}^{H} \boldsymbol{A}_{j}\right)^{-1} .
$$

We will see later that the matrix $S_{j}$ is used to determine $\boldsymbol{P}_{0}$, which is the focusing noise-free correlation matrix. In practice, $\boldsymbol{P}_{j}$ is directly computed from

$$
\boldsymbol{P}_{j}=\hat{\boldsymbol{R}}_{j}-\hat{\sigma}_{j}^{2} \boldsymbol{I}
$$

$P_{j}$ can be interpreted as the correlation matrix of the cleaned data. By the cleaned data, we mean the output of a preprocessing step that decreases the effect of the noise. The computational complexity of (25) is relatively low since the Lanczos algorithm [10] can be used to obtain a few of the smallest eigenvalues of $\hat{\boldsymbol{R}}_{j}$.

In general. the estimated source correlation matrix (24) may have negative eigenvalues. However, our simulation studies have shown that as far as the estimation of the DOA's is concerned, the TCT algorithm still can be used. As an alternative to (23) and to guarantee the nonnegativeness of the estimated source correlation matrix, the noise power can be estimated from

$$
\hat{\sigma}_{j}^{2}=\lambda_{p}\left(\hat{\boldsymbol{R}}_{j}\right)
$$

where $\lambda_{p}\left(\hat{\boldsymbol{R}}_{j}\right)$ is the smallest eigenvalue of $\hat{\boldsymbol{R}}_{j}$.

\section{B. Selecting the Focusing Subspace}

The noise-free focusing correlation matrix $P_{0}$ is a function of the DOA's, the frequency of focusing $f_{0}^{\prime}$, and the focusing source correlation matrix $\boldsymbol{S}_{()}$. The focusing DOA's are found using ordinary beamforming. We still have to choose $f_{0}$ and $\boldsymbol{S}_{0}$. The subspace fitting error is defined as

$$
\mathcal{E}=\sum_{j=1}^{J}\left\|\boldsymbol{P}_{0}-U_{j} \boldsymbol{P}_{j} \boldsymbol{U}_{j}^{H}\right\|^{2} .
$$

Using this error, we can select $f_{0}$ and $\boldsymbol{S}_{0}$ in two steps. In the first step, we choose the focusing source correlation matrix $\boldsymbol{S}_{0}$ as follows.

In an ideal case, all the transformed location matrices superimpose on $\boldsymbol{A}_{(1)}$. This case is called perfect focusing. In perfect focusing. the column vectors of the location matrix $\boldsymbol{A}$ are transformed to the corresponding columns of $\boldsymbol{A}_{0}$. i.e.

$$
\boldsymbol{A}_{0}=\boldsymbol{U}_{,} \boldsymbol{A}_{j} . \quad j=1 \ldots . .
$$

In such a case. the subspace fitting error can be shown to be

$$
\mathcal{E}=\sum_{j=1}^{J}\left\|\boldsymbol{A}_{0}\left(\boldsymbol{S}_{0}-\boldsymbol{S}_{j}\right) \boldsymbol{A}_{0}^{H}\right\|^{2} .
$$


Minimization of (29) for $S_{0}$ gives

$$
\boldsymbol{S}_{0}=\frac{1}{J} \sum_{j=1}^{J} \boldsymbol{S}_{j}
$$

The estimate of the $S_{j}$. which is given by (24), can be used in (30).

In practice, if the transformation is constrained to be unitary, perfect focusing cannot be obtained, but since the transformed location matrices are close to the focusing location matrix, the same focusing source correlation matrix (30) can be used in general case. The focusing source correlation matrix (30) has yet another important property. For coherent sources, the estimated source correlation matrix (24) might be singular. However, the average $S_{0}$ is full rank. Hence, (30) removes the coherence by smoothing the spectrum of the source signals. With this averaging, the TCT algorithm can be applied to coherent cases.

As seen from (20), the error of focusing is a function of $f_{0}$. To mininize this error, a suitable selection of the focusing frequency is needed. We seek a frequency $f_{0}$ that solves

$$
\begin{aligned}
& \mathcal{E}=\min _{j_{0}} \operatorname{mini1}_{\mathrm{U}_{i}} \sum_{j=1}^{I}\left\|P_{0}-U_{j} \boldsymbol{P}_{,} U_{j}^{H}\right\|^{2} \\
& \text { s.t. } \boldsymbol{U}_{i}^{H} \boldsymbol{U}_{i}=\boldsymbol{I}, \quad i=1 \ldots, J \text {. }
\end{aligned}
$$

For a fixed $\boldsymbol{P}_{0}$, the transformation matrices $\boldsymbol{U}_{j}$ are obtained from (21). By using these matrices in (31), the focusing error is given by

$$
\mathcal{E}=\sum_{j=1}^{J}\left[\left\|\boldsymbol{P}_{0}\right\|^{2}+\left\|\boldsymbol{P}_{j i}\right\|^{2}-2 \sum_{i=1}^{q} \sigma_{i}\left(\boldsymbol{P}_{0}\right) \sigma_{i}\left(\boldsymbol{P}_{j}\right)\right] .
$$

Since $\boldsymbol{P}_{j}$ 's are independent of the focusing frequency, $f_{0}$ can be determined from

$$
\underset{f_{0}}{\min } \sum_{j=1}^{I}\left[\sum_{i=1}^{q} \sigma_{i}^{2}\left(\boldsymbol{P}_{0}\right)-2 \sum_{i=1}^{q} \sigma_{i}\left(\boldsymbol{P}_{0}\right) \sigma_{i}\left(\boldsymbol{P}_{j}\right)\right] \text {. }
$$

To select the best focusing frequency, we first find the singular values of the optimum focusing subspace. Next, using these values, the focusing frequency is selected. Define

$$
\mu_{i}=\sum_{j=1}^{J} \sigma_{i}\left(\boldsymbol{P}_{j}\right)
$$

Using this definition, the criterion (33) is represented as

$$
\mathcal{E}=\min _{f_{0}} \sum_{i=1}^{q}\left[J \sigma_{i}^{2}\left(\boldsymbol{P}_{0}\right)-2 \mu_{i} \sigma_{i}\left(\boldsymbol{P}_{0}\right)\right]
$$

The minimum of (35) is achieved when

$$
\sigma_{i}\left(\boldsymbol{P}_{0}\right)=\frac{\mu_{i}}{J}, \quad i=1, \ldots, q .
$$

Due to structural constraint on $\boldsymbol{P}_{0}$, in general. (36) is not attainable. Instead, we perform

$$
\operatorname{mini}_{f_{i}} \sum_{i=1}^{\underline{Z}}\left|\sigma_{i}\left(\boldsymbol{P}_{0}\right)-\frac{\mu_{i}}{J}\right|^{2}
$$

This is a one-variable optimization problem, and a search procedure can be applied to find the minimum point. In practice, it is sometimes convenient to choose a predefined frequency such as the center frequency of the spectrum for focusing. However, to improve the performance, a focusing frequency that produces the smallest error should be selected.

\section{The TCT Algorithm}

The TCT algorithm is summarized as follows:

1) Use an ordinary beamformer to scan the space and find an initial estimate of the DOA's.

2) Apply a DFT to the array output to sample the spectrum of data.

3) Form $A_{j}$ and $S_{j}$ using the results of the preprocessing step and (24).

4) Average the source correlation matrices to obtain $S_{0}$ as in $(30)$.

5) Find $P_{0}=A_{0} S_{0} A_{0}^{H}$ and the $P_{j}$ 's using (25).

6) Determine the unitary transformation matrices (21).

7) Multiply these matrices by the sample correlation matrices, and average the results.

8) Use AIC, MDL [11], or PSC [12] to find the true number of sources.

9) Apply MUSIC or any other high-resolution spectral estimation method to find the DOA's.

10) To improve the performance, iterate steps 3 to 9.

Comparison of the new algorithm with the CSM method shows that the second part of Step 3 and Steps 4 and 5 do not have counterparts in CSM. The presence of these steps in TC.T increases the complexity of computation. The increase in the computation is due to three parts:

i) estimating the noise power in each frequency bin

ii) estimating the source correlation matrix from (24)

iii) forming the focusing correlation matrix $\boldsymbol{P}_{0}=$ $A_{0} S_{0} A_{0]}^{H}$.

To estimate the noise power for each frequency bin, we only need to compute a few of the smallest eigenvalues of the sample correlation matrix. To find those eigenvalues, the Lanczos algorithm [10] that converges in $O\left(p^{2}\right)$ flops for processing can be used. If we use (26) for noise power estimation, only one eigenvalue needs to be estimated.

The source correlation matrix at the $j$ th frequency bin is found from (24), where by defining $B_{j}=\left(A_{j}^{H} A_{j}\right)^{-1} A_{j}^{H}$ can be written as

$$
\hat{\boldsymbol{S}}_{j}=\boldsymbol{B}_{j}\left[\hat{\boldsymbol{R}}_{j}-\hat{\sigma}_{j}^{2} \Gamma\right] \boldsymbol{B}_{j}^{H}
$$

The computation of $B_{j}$ requires inverting $\left(A_{j}^{H} A_{j}\right)$ and nultiplying it by $\boldsymbol{A}_{j}^{H}$. Since $\left(\boldsymbol{A}_{j}^{H} \boldsymbol{A}_{j}\right)$ is a $q \times q$ Toeplitz matrix, it can be inverted in $O\left(q^{2}\right)$ flops [10]. Using $\boldsymbol{B}_{j}$, the source correlation matrix $\hat{S}_{j}$ is obtained with two matrix multiplications. The focusing correlation matrix $\boldsymbol{P}_{0}$ can also be formed by two matrix multiplications. It should be noted that the increase of the computational complexity is usually small compared with the load of Step 6.

Another difference in the computational complexity of the Iwo methods appears at Step 6, where an eigenvalue decon- 
position is performed. To find the unitary matrices in the CSM algorithm, $J$ singular value decompositions are needed. However, in TCT, $(J+1)$ eigenvalue decompositions of Hermitian matrices are required. This should be a smaller computational load.

\section{THE ERror of Transformation}

In this section, we compare the error of transformation for the CSM and TCT algorithms. There are two sources of error for the transformation: the error due to noisy observation and the error of transformation. The concern of the present section is the latter. We consider a noise-free environment where the error is only duc to the focusing procedure.

It is important to note that simply aligning the subspaces at different frequency bins does not result in a good estimate of the DOA's. The subspaces might be twisted in the process of focusing, causing an augmentation of the noise in some directions. This in turn reduces the focusing SNR and can result in a biased estimation of DOA's. To prevent warping of the subspaces, we can use unitary matrices for focusing. However, using unitary transformation matrices for focusing does not necessarily produce an unbiased estimate. Furthermore, there is no unique solution for the unitary focusing matrices. Here, we define an error of focusing that can be used as a comparison measure between different focusing methods.

Since the objective is to transform the noise-free correlation matrix at each frequency bin to the focusing noise-free correlation matrix, the subspace fitting error is given by

$$
\mathcal{E}=\left\|\boldsymbol{P}_{0}-\boldsymbol{W}_{j} \boldsymbol{P}_{j} \boldsymbol{W}_{i}^{H}\right\|^{2}
$$

where $W_{j}$ is the focusing matrix. The error of transformation for the TCT algorithm can be obtained by substituting $U_{j}$ in (39), which simplifies to

$$
\mathcal{E}_{\mathrm{TCT}}=\left\|\boldsymbol{P}_{0}\right\|^{2}+\left\|\boldsymbol{P}_{j}\right\|^{2}-2 \sum_{i=1}^{4} \sigma_{i}\left(\boldsymbol{P}_{0}\right) \sigma_{i}\left(\boldsymbol{P}_{i}\right) .
$$

The error of transformation for the CSM algorithm is represented by

$$
\mathcal{E}_{\mathrm{CSM}}=\left\|\boldsymbol{P}_{0}\right\|^{2}+\|\left.\boldsymbol{P}_{j}\right|^{2}-2 \Re \operatorname{Tr}\left(\boldsymbol{P}_{\| 1} \boldsymbol{T}_{j} \boldsymbol{P}_{j} \boldsymbol{T}_{j}^{H}\right)
$$

where $\boldsymbol{T}_{j}$ is the focusing matrix given by $(12)$. It is possible to show that the error of transformation for the CSM algorithm is given by

$$
\mathcal{E}_{\mathrm{CSII}}=\left\|\boldsymbol{P}_{i}\right\|^{2}+\left\|\boldsymbol{P}_{j}\right\|^{2}-2 \sum_{i=1}^{q} \sigma_{i}\left(\boldsymbol{P}_{0} \boldsymbol{T}_{j} \boldsymbol{P}_{j} \boldsymbol{T}_{j}^{H}\right) .
$$

Using Lemma 1. this error can be written as

$$
\begin{aligned}
\mathcal{E}_{\text {CSM }} & \geq\left\|\boldsymbol{P}_{0}\right\|^{2}+\left\|\boldsymbol{P}_{j}\right\|^{2}-2 \sum_{i=1}^{q} \sigma_{i}\left(\boldsymbol{P}_{0}\right) \sigma_{i}\left(\boldsymbol{T}_{j} \boldsymbol{P}_{j} \boldsymbol{T}_{j}^{H}\right)(43) \\
& =\left\|\boldsymbol{P}_{10}\right\|^{2}+\left\|\boldsymbol{P}_{j}\right\|^{2}-2 \sum_{i=1}^{q} \sigma_{i}\left(\boldsymbol{P}_{0}\right) \sigma_{i}\left(\boldsymbol{P}_{j}\right) \\
& =\mathcal{E}_{\mathrm{T} C \mathrm{~T}}
\end{aligned}
$$

where we have used the property that the matrices related with the similarity transformation have the same eigenvalues
[8]. Thus, the error of transformation for the TCT algorithm is always smaller than that for CSM.

\section{V1. Eigenvalles of the Universal FOCUSED CORRELATION MATRIX}

One of the major drawbacks of the CSM algorithm is the asymptotic bias of the peaks. It has been shown that the CSM a: gorithm generates an estimate of DOA that is asymptotically biased [7]. The bias increases with the bandwidth of processing and deviation of the focusing angles from the true DOA's. In this section, we study the eigenvalues of the universal focused correlation matrix of the two methods: CSM and TCT. Using the eigenvalues of the universal focused correlation matrix, we show that the signal power in the CSM algorithm is extended into the noise subspace. This extension acts as a spatially colored noise with an unknown correlation matrix that produces biased estimates of the DOA's.

\section{A. Analytical Study}

To study the mechanism that generates the asymptotic bias, a noise-free environment is considered. In such a case, the correlation matrix $\hat{\boldsymbol{R}}_{j}$ is equal to the array noise-free correlation matrix $P_{j}$. The universal focused correlation matrix is

$$
\begin{aligned}
\boldsymbol{R}_{\mathrm{CS} \mathrm{Y}} & =\frac{1}{.} \sum_{j=1}^{J} \boldsymbol{T}_{j} \boldsymbol{P}_{j} \boldsymbol{T}_{j}^{H} \\
& =\frac{1}{J} \sum_{j=1}^{J} V_{i} W_{j}^{H} A_{j} S_{i} A_{j}^{H} W_{j} V_{j}^{H} .
\end{aligned}
$$

The focused correlation matrix is a function of the focusing angles and the bandwidth of sources. In general, $R_{\mathrm{CSNI}}$ is full rank and has nonzero eigenvalues in the noise subspace. In other words, the received power is distributed in a $p$ dimensional space. The components of the signal that diffuse into the noise subspace act as a nonwhite noise with an unknown correlation matrix. The MUSIC algorithm that operates on $R_{C 5 M}$ will provide biased estimates of the DOA's if the spatial noise structure is unknown.

For TCT, the universal focused correlation matrix is given by

$$
\begin{aligned}
\boldsymbol{R}_{\mathrm{TCT}} & =\frac{1}{J} \sum_{j=1}^{J} \boldsymbol{U}_{j} \boldsymbol{P}_{j} \boldsymbol{U}_{j}^{H} \\
& =\frac{1}{J} \sum_{j=1}^{J} \boldsymbol{X}_{0} \boldsymbol{X}_{j}^{H} \boldsymbol{P}_{j} \boldsymbol{X}_{j} \boldsymbol{X}_{0}^{H}
\end{aligned}
$$

where (21) has been used to compute $U_{j}$. Suppose that the diagonal matrices of the eigenvalues of $\boldsymbol{P}_{j}, j=1 \ldots \ldots J$ are shown by $\Gamma_{j} . j=1 \ldots \ldots$. Then, for any focusing angle, (47) simplities to

$$
\begin{aligned}
\boldsymbol{R}_{\mathrm{TC}\ulcorner} & =\frac{1}{J} \sum_{j=1}^{J} \boldsymbol{X}_{0} \boldsymbol{\Gamma}_{j} \boldsymbol{X}_{0}^{H} \\
& =\boldsymbol{X}_{0} \boldsymbol{\Gamma}_{0} \boldsymbol{X}_{0}^{H}
\end{aligned}
$$


where $\Gamma_{0}=\frac{1}{J} \sum_{j=1}^{J} \Gamma_{j}$. Since $\boldsymbol{P}_{j}$ is computed directly from the sample correlation matrix using (25), the diagonal matrix $\Gamma_{0}$ is independent of the pre-estimates of DOA. Note that each diagonal matrix $\Gamma_{j}$ has only $q$ nonzero entries, which in turn implies that $\Gamma_{0}$ has only $q$ nonzero components. The matrix $X_{0}$ is orthonormal, and hence, (48) is an eigenvalue decomposition of $\boldsymbol{R}_{\mathrm{TCT}}$. It is concluded that in TCT, the transformed subspaces for different frequencies are aligned, and the eigenvalues at the noise subspace are zero. In other words, the focused correlation matrix $\boldsymbol{R}_{\mathrm{IC}: \mathrm{T}}$ has eigenvalues in a $q$-dimensional subspace. This is an important property of the TCT algorithm that makes it capable of providing unbiased estimates of the DOA's.

It is useful to compare (48) with perfect focusing. In perfect focusing, the noise-free focused correlation matrix is $\boldsymbol{A}_{0} \boldsymbol{R}_{s} \boldsymbol{A}_{0}^{H}$, where $\boldsymbol{R}_{s}$ is defined in (8). The perfect focusing can be obtained using the transformation matrices (4). These transformation matrices are not unitary. In general, it is not possible to establish perfect focusing of the location matrices through unitary transformations. In (48), perfect focusing is achieved by applying the transformation to the eigenvectors of the correlation matrices. Since the eigenvectors form an orthonormal basis, it is always possible to use a unitary transformation to transfer them into another orthonormal basis. Note that the true DOA implicitly effects the selection of the focusing subspace through the estimation of $S_{j}$. Thus, assignment of the orthonormal basis for the focusing subspace is implied by the true DOA's.

\section{B. Experimental Results}

We present the results of a computer simulation to study the eigenvalues of the universal correlation matrix of the two methods. A configuration with four equipower wideband signals arriving at a linear array of 16 sensors in a noise-free environment is considered. The true DOA's are $8,13,33$, and $37^{\circ}$. The spectrum of the signals is flat with $40 \%$ relative bandwidth. The initial DOA's are taken at 6.7, $10.5,14.3,31,35$, and $39^{\circ}$. The output of the sensors is decomposed into 50 snapshots with each snapshot containing 64 samples. An FFT algorithm is used in each snapshot to sample the frequency spectrum of the signals at 33 equispaced points. We applied the CSM and TCT algorithms to obtain the focusing matrices. The eigenvalues of the corresponding matrices are tabulated in Table I. It is seen that $\boldsymbol{R}_{\mathrm{CSMI}}$ has nonzero eigenvalues in the noise subspace due to signal diffusion. Since the focusing matrices $T$, and $U_{j}$ are unitary, the trace of $R_{\mathrm{CSM}}$ is equal to the trace of $R_{\mathrm{TCT}}$. This means that the summation of eigenvalues in Table I is identical for each matrix. This suggests that the energy of the signals after focusing is identical for the two methods. However, the TCT method condenses the total received energy in a q-dimensional subspace and, hence, improves the performance.

\section{THE BIAS OF Estimation}

One of the major motivations for introducing the TCT algorithm is to reduce the asymptotic bias of the peaks in CSM. It is important to note that the MUSIC algorithm is intrinsically
TABLE I

Eigenvalues df the Correlation Matrices $\boldsymbol{R}_{\text {CSM ANd }} \boldsymbol{R}_{\text {TCt }}$ for a Configuration of Four Wideband Solres. THE SOUtrces HaVE $40 \%$ Relative Bandwidth and ARE ARRIVING FROM the ANGLEs '8, 13, 33, AND $37^{\circ}$ at a Linear array of 16 Sensors in a Noise-Free Evvironment,

\begin{tabular}{crr}
\hline eigenvalues & $\mathbf{R}_{\text {CSM }}$ & $\mathbf{R}_{\text {TCT }}$ \\
\hline$\lambda_{1}$ & 900.27 & 918.76 \\
$\lambda_{2}$ & 806.70 & 808.09 \\
$\lambda_{3}$ & 241.68 & 244.14 \\
$\lambda_{4}$ & 155.39 & 140.99 \\
$\lambda_{5}$ & 4.39 & 0.00 \\
$\lambda_{6}$ & 1.35 & 0.00 \\
$\lambda_{7}$ & 0.40 & 0.00 \\
$\lambda_{8}$ & 0.34 & 0.00 \\
$\lambda_{9}$ & 0.31 & 0.00 \\
$\lambda_{10}$ & 0.27 & 0.00 \\
$\lambda_{11}$ & 0.22 & 0.00 \\
$\lambda_{12}$ & 0.19 & 0.00 \\
$\lambda_{13}$ & 0.15 & 0.00 \\
$\lambda_{14}$ & 0.12 & 0.00 \\
$\lambda_{15}$ & 0.09 & 0.00 \\
$\lambda_{16}$ & 0.07 & 0.00 \\
\hline
\end{tabular}

unbiased. The bias in CSM is introduced by focusing. This implies that one can reduce the bias of estimation with a proper selection of the focusing method. In the sequel, we will discuss this issue and show that TCT can asymptotically generate an unbiased estimate of the DOA's. We will also generalize the work of Swingler and Krolik [13]. They showed that for a single-source scenario, it is possible to have an unbiased estimate, provided that the focusing frequency is chosen at the centroid of the source spectrum. Here, we show that for a multisource case, the bias is eliminated if the focusing correlation matrix for the true value of the DOA's is the average of the transformed correlation matrices. Since the bias of estimation is independent of the noise, in the rest of this section a noise-free environment is considered.

The noise-free universal focused correlation matrix is shown as

$$
\boldsymbol{R}=\frac{1}{J} \sum_{j=1}^{I} \boldsymbol{W}_{j} \boldsymbol{P}_{j} \boldsymbol{W}_{j}^{H}
$$

where $\boldsymbol{W}_{j}$ is the focusing matrix for the $j$ th frequency bin. A sufficient condition for unbiased estimation is given by the following lemma.

Lemma 2: Any focusing method that satisfies

$$
\overline{\boldsymbol{A}}_{0} \boldsymbol{S}_{0} \overline{\boldsymbol{A}}_{\mathrm{I}}^{H}=\frac{1}{7} \sum_{j=1}^{J} \boldsymbol{W}_{j} \boldsymbol{P}_{j} \boldsymbol{W}_{j}^{H}
$$

where $\boldsymbol{S}_{0}$ is a nonsingular, Hermitian, positive-definite matrix, and $\overline{\boldsymbol{A}}_{0}$ is the true location matrix at the focusing frequency, produces an unbiased extimate of the DOA's.

Proof: Since $\boldsymbol{S}_{0}$ is nonsingular,

$$
\operatorname{Span}\left\{\overline{\boldsymbol{A}}_{0}\right\}=\operatorname{Span}\left\{\boldsymbol{V}_{q}\right\}
$$

where $V_{q}$ is the matrix of the $q$ largest eigenvectors of $\boldsymbol{R}$. The MUSIC estimator intersects the subspace spanned by the 
$q$ largest eigenvectors of the correlation matrix with the array manifold. Therefore, if $(50)$ is satisfied, the estimation will be unbiased.

It is important to notice that (50) is a general condition for unbiased estimation regardless of the method that has been applied for focusing. In [13], Swingler and Krolik showed that for a single source scenario, an unbiased estimation of the DOA is possible if the centroid of the frequency spectrum is chosen as the focusing frequency. Lemma 2 shows that in a multisource case, the focusing correlation matrix should be the average of the focused correlation matrices for unbiased estimation.

For further discussion of the bias generating mechanism, we consider the special case of perfect focusing. In perfect focusing, the transformed correlation matrices $U_{j} \boldsymbol{A}_{j}$ are superimposed on $\boldsymbol{A}_{1}$. In such a case, the focusing correlation matrix is an average of the correlation matrices at the frequency bins, and the following equality is satisfied:

$$
A_{0} S_{0} A_{0}^{H}=\frac{1}{j} \sum_{j=1}^{I} U_{j} A_{j} S_{j} A_{j}^{H} U_{j}^{H}
$$

As noted earlier, for any $\hat{\theta}_{i}$ estimated by the preprocessing step, two more focusing angles are added in the vicinity of $\hat{\theta}_{i}$. These angles determine an interval on the array manifold. If this interval is sinall compared with the curvature of the array manifold at all the points in that interval, it is transferred to a corresponding interval on the array manifold at the focusing frequency $f_{0}$. This is a consequence of the continuity of the array manifold and the unitary transformation. Thus, in perfect focusing, the location yectors of each frequency bin that are located at the true DOA are transferred to the corresponding vectors at the focusing manifold. It is seen that in such a case, the estimation of DOA can be unbiased.

In practice, perfect focusing is not possible. The transformed matrices are clustered around $\boldsymbol{A}_{()}$. However, as far as the equality (50) is satisfied for the true DOA, estimation could be unbiased. It is straightforward to show that the TCT algorithm forms a very good approximation of (50). Taking the gradient of (31) with respect to $P_{0}$ and equating to zero proves that the minimum of (31) is achieved if and only if $P_{0}$ is the average of the matrices $U_{j} P_{j} U_{j}^{H} \cdot j=1 \ldots \ldots$. . The TCT algorithm is based on the minimization of (31), which means that it places the focusing correlation matrix at the average of the matrices $U_{i l} P_{j} U_{j}^{H H} \cdot j=1 \ldots \ldots$. . However, because of unknown DOA's and structural constraints on the correlation matrix, it is not possible to satisfy $(50)$ with equality. The TCT algorithm provides a close approximation to $(50)$, which explains its capability to provide asymptotically unbiased estimates.

\section{The Effect of NoIse on Estimation}

It is possible to show that the sample correlation matrices of the two methods (CSM and TCT) are Wishart distributed [14] with $J N$ degrees of freedom with the correlation matrices $\boldsymbol{R}_{\mathrm{CSM}}$ and $\boldsymbol{R}_{\mathrm{TCT}}$, respectively. However, simply considering the degrees of freedom is not sufficient for comparison since any transformation of the signal subspaces results in a Wishart distributed correlation matrix with $.7 N$ degrees of freedom. The important factor is how the transformed observation vectors are distributed in the $p$-dimensional subspace. In this section, we will show that the variance of the noise is smaller for TCT, which results in a better estimate of the DOA's.

The observation can be considered to be a p-dimensional signal vector in the $q$-dimensional signal subspace perturbed by a $p$-dimensional noise vector. It is important to note that the noise component in the signal subspace has no effect in the estimation process since the MUSIC algorithm estimates the DOA's by intersecting the signal subspace with the array manifold. In other words, if the noise is restricted to the signal subspace, there will be no error in the estimation. However, the power of the noise in the noise subspace is very important in introducing error in the estimation. The effect of the orthogonal noise can be discussed based on the theory of generalized variance [14]. The generalized variance of a multivariate random vector is defined as the determinant of the correlation matrix. The generalized variance is a metric for the spread of the observation and is equal to the sum of squares of the volumes of all different parallelotopes formed by using any $p$ observation vectors as the principal edges [14].

In a noise-free environment, the observation vectors are constrained to the signal subspace, and all the parallelotopes have zero volume in the $p$-dimensional observation space. Thus, the generalized variance in such a case is zero. This is the key point to the estimation of the DOA's without error. When the noise is introduced in the system, the observation vectors are expanded into the noise subspace. Extension of the signal vectors into the noise subspace results in a nonzero yolume and, hence, a nonzero generalized variance. Thus, for a noisy environment, the estimation of the DOA's is usually yielded with error. The sinaller the volume of the observation vectors, the better the estimate of the DOA's. Therefore, a small generalized variance results in a small variance of estimation. In what follows, we show that the generalized variance of the TCT algorithm is smaller than that for CSM.

Consider the following maximization problem:

$$
\begin{gathered}
\operatorname{Ilax}|\boldsymbol{R}| \quad \text { s.t. } \operatorname{tr} \boldsymbol{R}=\mathrm{constant} \\
\boldsymbol{R}>0
\end{gathered}
$$

where $|\cdot|$ stands for the determinant of a matrix, and $\boldsymbol{R}>0$ means that the matrix is positive definite. It is known that the maximum of $|\boldsymbol{R}|$ is obtained if all the eigenvalues are equal. The maximum corresponds to an equilibrium point where the energy is equally distributed in all dimensions. Assume that the eigenvalues are components of a vector. The vector of the eigenvalues that satisfy the constraints of (53) are located in a portion of a hyperplane that is cut by the positive quadrant. The equilibriun point that is the solution of $(53)$ is at the centroid of this region. The maximization (53) is a convex problem. Thus, the farther we are from the equilibrium point, the smaller $|R|$ will be.

Now, we compare the determinant of $\boldsymbol{R}_{\mathrm{CSMI}}$ and $\boldsymbol{R}_{\mathrm{TCT}}$. Note that both $\boldsymbol{R}_{\mathrm{CSMI}}$ and $\boldsymbol{R}_{\mathrm{TCT}}$ are positive definite matrices 
with the trace given by

$$
\operatorname{tr} \boldsymbol{R}_{\mathrm{CSM}}=\operatorname{tr} \boldsymbol{R}_{\mathrm{TCT}}=\frac{1}{J} \sum_{j=1}^{J} \operatorname{tr} \hat{\boldsymbol{R}}_{j}
$$

which is a constant independent of the focusing method. Thus, they satisfy the constraints of (53). Note that requiring the trace to be constant assures that the energy is not lost during the focusing process. We have shown in Section VI that in the CSM algorithm, the signal energy is extended into the noise subspace. In other words, the eigenvalues of $\boldsymbol{R}_{\mathrm{TCT}}$ in the noise subspace are smaller than the eigenvalues of the $\boldsymbol{R}_{\mathrm{CSM}}$, whereas the sum of eigenvalues for the two methods are equal. Thus, the vector of eigenvalues for the TCT algorithm is farther from the equilibrium point in the maxinization (53) than that for CSM. This means that the universal focused correlation matrix of the TCT algorithm has smaller determinant or generalized variance. The smaller generalized variance of the TCT algorithm results in a smaller variance in the estimation of the DOA's.

\section{iX. Performance Comparison}

Recently, Doron and Weiss [15] introduced a method for wide-band array processing using signal subspace transformation (SST). The focusing matrix in their method is found from minimization of $\left\|A_{0} D_{0} A_{l\}}^{H}-T_{j} A_{j} D_{j} A_{j}^{H} T_{j}^{H}\right\|$, where $D_{0}$ and $D_{j}$ are any Hermitian positive definite matrices. They used identity matrices for $D_{0}$ and $D_{j}$ in their simulation. The results of the simulation shows that the method is biased. There are two major differences between the TCT and SST algorithms. First, in TCT, the average of the estimated source correlation matrices at the frequeticy bins is chosen as the focusing source correlation matrix in place of $D_{0}$. Second, instead of $\boldsymbol{A}_{j} \boldsymbol{D}_{j} \boldsymbol{A}_{j}^{H}$, the estimated noise-free correlation matrices $\boldsymbol{P}_{j}$ are used for focusing matrix determination. Note that in $A_{j} D_{j} A_{j}^{H}$, the estiniated DOA's are utilized; however, for $\boldsymbol{P}_{j}$, the true DOA"s are implicitly used.

There is also a difference between the model equations of the CSM and TCT algorithms. In CSM, the focusing model equations are $T_{j} A_{j}=A_{0}$. The solution to this equation is not unique and might be singular. It has been shown [7] that from an estimation point of view, unitary transformations of signal subspaces are the most effective focusing methods for direction finding. In contrast, in TCT, the model equations are $\boldsymbol{U}_{j} \boldsymbol{P}_{j} \boldsymbol{U}_{j}^{H}=\boldsymbol{P}_{0}$, where $\boldsymbol{P}_{j}=\hat{\boldsymbol{R}}_{j}-\hat{\sigma}_{j}^{2} \boldsymbol{I}$, and $\boldsymbol{P}_{0}=\boldsymbol{A}_{0} \boldsymbol{S}_{0} \boldsymbol{A}_{0}^{H}$, with $S_{0}$ given by (30). Note that the noise-free correlation matrix $P_{j}$ is directly estimated from the data, and the preestimate of the DOA's is not used in its determination.

\section{A. Simulation Results}

Here, we present the simulation results for two DOA estimation scenarios. In the first example, a configuration with two sources is considered. For this example, we have compared the bias, the resolution threshold, and the spatial spectrum of the MUSIC algorithm of the two methods (CSM and TCT). The second example is a multigroup DOA estimation problem with the angles taken from [7]. We have found the bias of
TABLE II

E5TIVATION Results for the FIRST EXAMPLE

\begin{tabular}{|c|c|c|c|c|c|c|}
\hline \multirow{2}{*}{$\begin{array}{c}\mathrm{BW}=0.4 \\
f_{0}\end{array}$} & \multicolumn{3}{|c|}{ CSM } & \multicolumn{3}{|c|}{ TCT } \\
\hline & 11 & 13 & bias & 11 & 13 & bias \\
\hline 0.8 & 11.89 & 12.13 & 1.24 & 11.00 & 13.00 & 0.00 \\
\hline 0.9 & 11.25 & 12.75 & 0.35 & 11.00 & 13.00 & 0.00 \\
\hline 1.0 & 11.01 & 12.99 & 0.01 & 11.00 & 13.00 & 0.00 \\
\hline 1.1 & 10.88 & 13.12 & 0.17 & 11.00 & 13.00 & 0.00 \\
\hline 1.2 & 10.78 & 13.22 & 0.31 & 11.00 & 13.00 & 0.00 \\
\hline$\overline{\mathrm{BW}}=1.0$ & \multicolumn{3}{|c|}{$\mathrm{CSM}$} & \multicolumn{3}{|c|}{ TCT } \\
\hline$f_{0}$ & 11 & 13 & bias & 11 & 13 & bias \\
\hline 0.8 & - & 12.01 & - & 11.01 & 12.99 & 0.01 \\
\hline 0.9 & 11.42 & 12.58 & 0.59 & 11.00 & 13.00 & 0.00 \\
\hline 1.0 & 11.12 & 12.88 & 0.17 & 11.00 & 13.00 & 0.00 \\
\hline 1.1 & 10.95 & 13.05 & 0.07 & 11.00 & 13.00 & 0.00 \\
\hline 1.2 & 10.84 & 13.16 & 0.23 & 11.00 & 13.00 & 0.00 \\
\hline
\end{tabular}

the estimation of the CSM and TCT algorithms and compared them. We have also shown that the TCT algorithm can locate coherent sources.

Two Sources: In the first example, we investigate a configuration with two equipower uncorrelated sources impinging from the angles 11 and $13^{\circ}$ off broadside. The SNR is $10 \mathrm{~dB}$. A linear array of eight sensors is used. The spacing between adjacent sensors is equal to half the wavelength at the center frequency. An ordinary beamformer gives a peak at $12^{\circ}$. Two extra focusing points are added at 9 and $15^{\circ}$. Sources are sampled with 33 frequency bins in the frequency domain. We imported the actual correlation matrix to the CSM and TCT algorithms and used the high-resolution MUSIC algorithm for DOA estimation. The results of the estimation for 40 and $100 \%$ bandwidth and for different focusing frequencies are given in Table II. The bias columns in this table are the Euclidean norm of the bias vectors. TCT does not have bias regardless of the bandwidth of processing.

For this example, we investigate the threshold SNR for the two methods. The output of each sensor is separated into 50 snapshots of 16 samples each. Then, an FFT algorithm is applied in each snapshot to sample the spectrum of the observation at nine points. To find the resolution threshold. 100 independent trials of the same scenario were run for each SNR. The number of times each algorithm resolved the sources was counted to estimate the probability of resolution. The sources were assunied to be resolved when two peaks in the spatial spectrum of the MUSIC algorithm were observed. Fig. 1 shows the probability of the resolution for the two methods. Two versions of the CSM algorithm are used here. By UCSM and DCSM, we mean unitary and diagonal versions of the CSM algorithm, respectively. For DCSM, the focusing angle is chosen at $12^{\circ}$. It is seen that TCT has a lower SNR threshold compared with the UCSM and DCSM algorithms.

We also examine the resolution capability of the two algorithms. We increase the number of sensors to 16 and consider a $40 \%$ relative bandwidth. It is assumed that only 20 snapshots of data are available. Again, at each snapshot, a 64-point DFT is applied to obtain 33 frequency samples in the frequency domain. The resolution criterion is defined as the difference 
TABLE III

Estimation Results for the Second Example. The Blank Entries Mean that CSM Did Not Resolwe the DoA

\begin{tabular}{|c|c|c|c|c|c|c|c|c|c|c|}
\hline \multirow{2}{*}{$\begin{array}{c}\mathrm{BW}=0.4 \\
f_{0}\end{array}$} & \multicolumn{5}{|c|}{ CSM } & \multicolumn{5}{|c|}{ TCT } \\
\hline & 8 & 13 & 33 & 37 & biss & 8 & 13 & 33 & 37 & bias \\
\hline 0.8 & - & 11.75 & 34.25 & $=$ & - & 8.03 & 13.03 & 32.98 & 36.98 & $0 . \overline{05}$ \\
\hline 0.9 & 8.42 & 12.55 & 33.90 & - & - & 8.01 & 13.01 & 32.98 & 36.98 & 0.03 \\
\hline 1.0 & 8.07 & 13.01 & 33.12 & 36.82 & 0.23 & 8.02 & 13.02 & 32.97 & 36.97 & 0.05 \\
\hline 1.1 & 7.87 & 13.21 & 32.53 & 37.35 & 0.64 & 8.07 & 13.06 & 32.92 & 36.91 & 0.15 \\
\hline 1.2 & 7.72 & 13.29 & 32.22 & 37.66 & 1.10 & 8.17 & 13.13 & 32.83 & 36.81 & 0.33 \\
\hline \multirow{2}{*}{$\begin{array}{c}\mathrm{BW}=1.0 \\
f_{0}\end{array}$} & \multicolumn{5}{|c|}{$\overline{\mathrm{CSM}}$} & \multicolumn{5}{|c|}{$\overline{\overline{T C T}}$} \\
\hline & 8 & 13 & 33 & $3 \bar{T}$ & bials & 8 & 13 & 33 & 37 & bias \\
\hline 0.8 & - & 11.17 & 34.22 & - & - & 8.04 & 13.08 & 32.86 & 36.88 & 0.20 \\
\hline 0.9 & - & 11.85 & 33.88 & - & - & 8.07 & 13.09 & 32.88 & 36.89 & 0.20 \\
\hline 1.0 & 8.25 & 12.53 & 33.58 & - & - & 8.10 & 13.10 & 32.87 & 36.87 & 0.23 \\
\hline 1.1 & 7.98 & 12.93 & 33.21 & 36.97 & 0.22 & 8.15 & 13.13 & 32.85 & 36.85 & 0.29 \\
\hline 1.2 & 7.85 & 13.13 & 32.78 & 37.44 & 0.53 & 8.22 & 13.19 & 32.72 & 36.71 & 0.50 \\
\hline
\end{tabular}

TABLE IV

ESTIMATION RESULTS FOR THE SECOND EXAYPLE AFTER TWO ITERATIONS

\begin{tabular}{|c|c|c|c|c|c|c|c|c|c|c|}
\hline \multirow{2}{*}{$\begin{array}{c}\mathrm{BW}=0.4 \\
f_{0}\end{array}$} & \multicolumn{5}{|c|}{$\overline{C S M}$} & \multicolumn{5}{|c|}{$\overline{\mathrm{TCT}}$} \\
\hline & 8 & 13 & 33 & 37 & bias & 8 & 13 & 33 & 37 & bias \\
\hline 0.8 & 8.04 & 12.99 & 33.03 & $\overline{36.90}$ & $0.1 \overline{1}$ & 8.00 & 13.00 & 33.00 & 37.00 & 0.00 \\
\hline 0.9 & 8.02 & 12.99 & 33.03 & 36.95 & 0.06 & 8.00 & 13.00 & 33.00 & 37.00 & 0.00 \\
\hline 1.0 & 7.99 & 12.99 & 33.04 & 37.03 & 0.05 & 8.00 & 13.00 & 33.00 & 37.00 & 0.00 \\
\hline 1.1 & 7.99 & 13.01 & 32.98 & 37.03 & 0.04 & 8.00 & 13.00 & 33.00 & 37.00 & 0.00 \\
\hline 1.2 & 7.98 & 13.03 & 32.94 & 37.03 & 0.08 & 8.00 & 13.00 & 33.00 & 37.00 & 0.00 \\
\hline
\end{tabular}

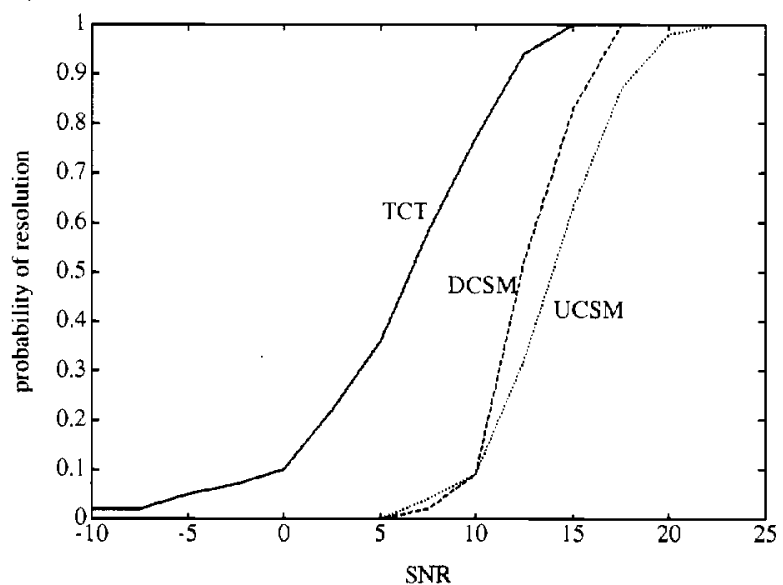

Fig. 1. Probability of resolution for two closely' separated sources using the TCT and CSM algorithms.

between the average of the spatial spectrum at the peak points in the MUSIC algorithm and the spatial spectrum in the valley [16]. It is measured on a decibel scale for different SNR's. The results are given in Fig. 2. As it is seen. the performance of TCT is about $6 \mathrm{~dB}$ better than that for CSM. The spatial spectra of the two methods are overlapped in Fig. 3 for comparison.

Four Sources: For the second configuration we investigate Example 1 in [7]. The same array has been used to estimate the DOA of four equipower uncorrelated sources impinging from $8,13,33$ and $37^{\circ}$. The bandwidth of the sources is equal to 40 and $100 \%$ of the center frequency in two different trials. The

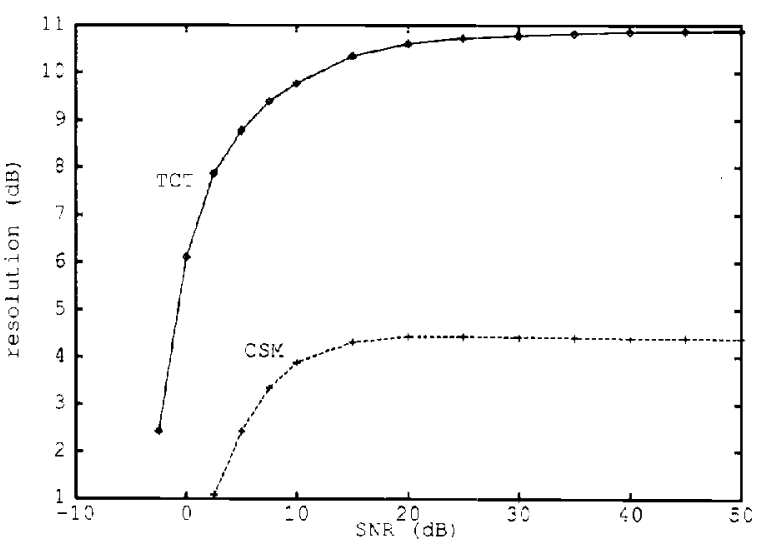

Fig. 2. Resolution compurison between the two atgorithms (TCT and CSM)

focusing angles are given by $6.7,10.5,14.3,31,35$, and $39^{\circ}$ The true cross correlation matrices are used for estimation. Table III presents the results of estimation for this example. Although TCT performs better, it is not unbiased. To improve the resolution and reduce or eliminate the bias, we can iterate the algorithm. We consider an array of 16 sensors with the same four signals as the second example. Application of the TCT method gives the estimates of the DOA's at 7.94, 13.03. 33.09 and $37.08^{\circ}$. We use the following focusing angles: 7 . $7.94,9,12,13.03,14,32,33.09,34,36,37.08$, and $38^{\circ}$. The results for the two methods are given in Table IV. As it is seen, the TCT algorithm outperforms CSM and removes the bias of the estimation. In general, to eliminate the bias, this procedure must be iterated several times. 


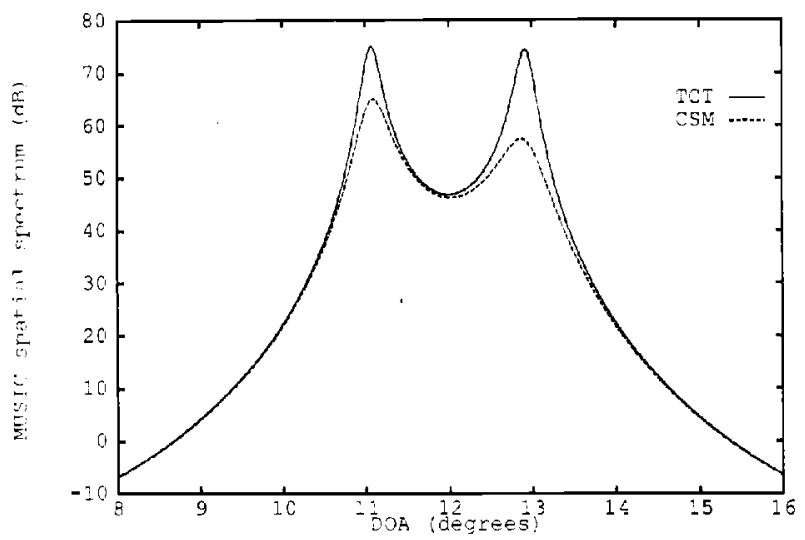

Fig. 3. MUSIC spatial spectrum for the two methods (TCT and CSM).

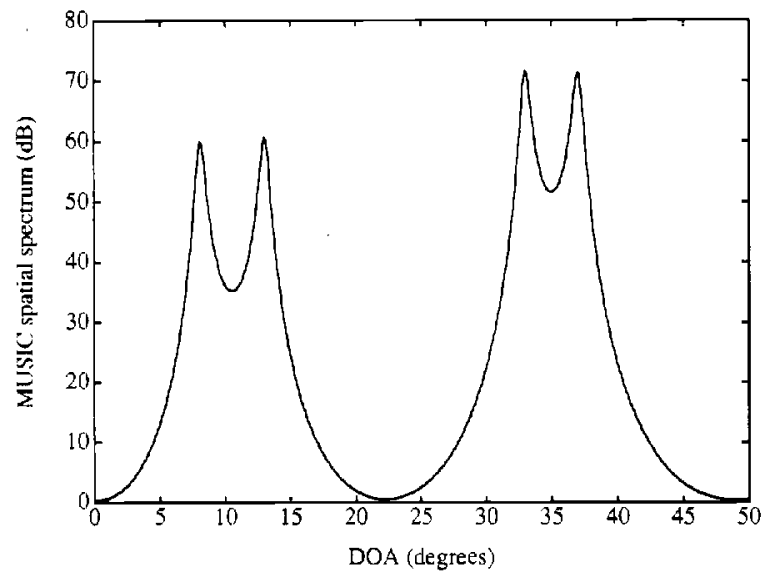

Fig. 4. MUSIC spatial spectrum for four sources at $8,13,33$, and $37^{\circ}$. The source at $13^{\circ}$ is a delayed version of the source at $8^{\circ}$.

We also investigate the capability of the TCT algorithm to resolve coherent sources. In Fig. 4, the MUSIC spatial spectrum for the coherent source scenario at $10 \mathrm{~dB}$ SNR is depicted. It is assumed that the source at $13^{\circ}$ is a delayed version of the source at $8^{\circ}$ with a one sampling time delay. As it is seen, the TCT algorithm resolves all the sources including the coherent ones.

To study the performance of the TCT algorithm for a limited number of observations, the same four sources are received by a linear array of 16 sensors. The observation interval is decomposed into 40 snapshots with each containing 32 samples. The focusing angles are taken at $6.7 .10 .5,14.3,31$, 35 , and $39^{\circ}$. The SNR is varied, and the bias and the variance are averaged over 100 independent trials. In Figs. 5 and 6 , the norm of the bias and the variance vectors for the two methods are compared. These examples show that the TCT algorithm has smaller bias and variance for a limited number of observations.

\section{SUMmarY}

In this paper, we have introduced a new method for localization of broadband signals using an array of sensors. Our

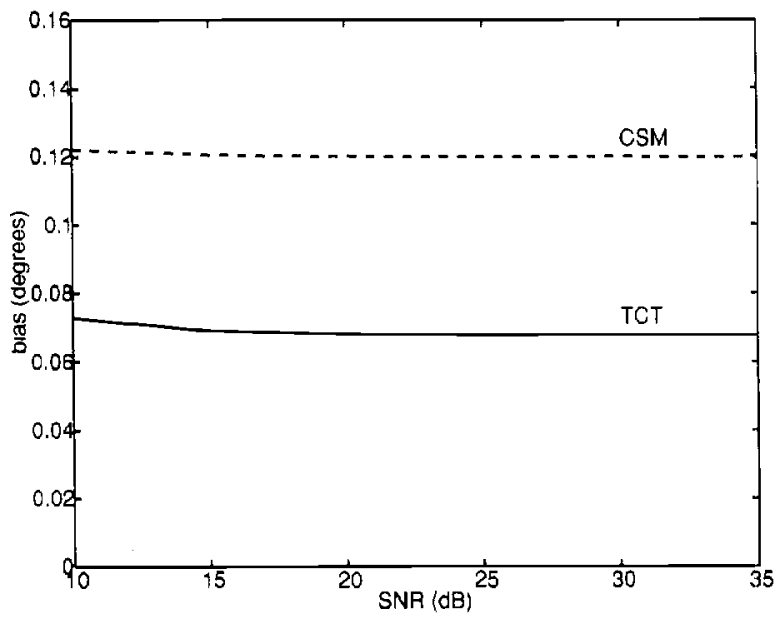

Fig. 5. Norm of the averaged bias vector for limited number of observations (40 snapshots of 32 samples) versus $S N R$ for a configuration with four uncorrelated sources.

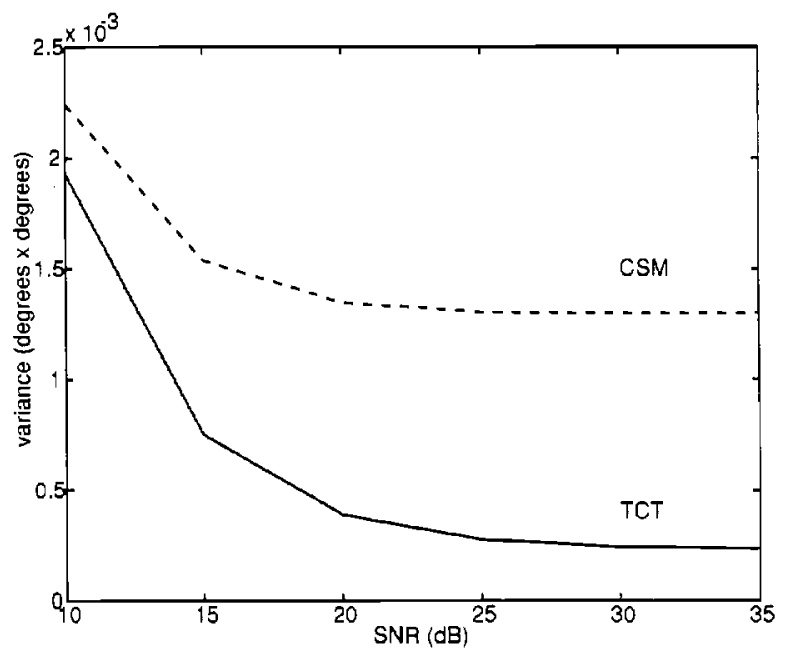

Fig. 6. Norm of the averaged variance vector for limited number of observations (40 snapshots of 32 samples) versus SNR for a configuration with foul" uncorrelaled sources.

method is based on the two-sided unitary transformation of the correlation matrices. The motivation for this work was to reduce the error of the subspace fitting and to remove the asymptotic bias of estimation that is involved in the CSM algorithm. The bias of estimation in CSM is a function of the focusing points and the bandwidth of processing. We have shown that the noise-free universal focused sample correlation matrix has nonzero eigenvalues in the noise subspace. This nonuniform expansion of the source energy into the noise subspace acts as a colored noise with an unknown correlation matrix. Since the form of the signal extension is unknown, the estimation will be biased in general. We have shown that the TCT algorithm does not suffer from this shortcoming. The noise-free universal focused correlation matrix of the TCT algorithm has q nonzero eigenvalues, and its columns 
span a $q$-dimensional subspace regardless of the processing bandwidth. With iterative use of the TCT algorithm, it is possible to coincide this $q$-dimensional subspace with the true signal subspace; hence, there is unbiased estimation. We have also shown that the generalized variance of the TCT algorithm is smaller than its counterpart for CSM. Thus, in a noisy situation. TCT can generate estimates of the DOA's with a smaller variance.

\section{APPENDIX A \\ Singular Values of a Matrix Product}

In this Appendix, we prove Lemma 1. We start by introducing a lemma from [8].

Lemma A.l: Let $\boldsymbol{A} . \boldsymbol{B} \in \boldsymbol{M}_{m \times n}$ (an $m \times n$ matrix), and $q=\min \{m, n\}$. Denote by $\sigma_{i}(\boldsymbol{A}), \sigma_{i}(\boldsymbol{B})$ and $\sigma_{i}\left(\boldsymbol{A} B^{H}\right)$. $i=1, \ldots, q$, the nonzero singular values of the corresponding matrices arranged in nonincreasing order. Then, for $U \in$ $M_{m \times m}, V \in M_{n \times n}$ being unitary

$$
\underset{\mathbf{U}, \mathbf{V}}{\max }\left\{\Re \operatorname{Rt} \boldsymbol{A} \boldsymbol{V}^{H} \boldsymbol{B}^{H} \boldsymbol{U}^{H}\right\}=\sum_{i=1}^{q} \sigma_{i}(\boldsymbol{A}) \sigma_{i}(\boldsymbol{B})
$$

We use Lemna A.1 to prove Lemma 1. Suppose that the singular value decomposition of the matrices $A$ and $B$ are given by

$$
\begin{aligned}
& A=E \Lambda_{u} F^{H} \\
& B=X \Lambda_{b} Y^{H}
\end{aligned}
$$

Then

$$
\begin{aligned}
\sum_{i=1}^{q} \sigma_{i}\left(\boldsymbol{A} \boldsymbol{B}^{H}\right) & =\Re \operatorname{tr}\left(\boldsymbol{A} \boldsymbol{B}^{H}\right) \\
& =\Re \operatorname{tr}\left(\boldsymbol{\Lambda}_{a} \boldsymbol{F}^{H} \boldsymbol{Y} \boldsymbol{\Lambda}_{b} \boldsymbol{X}^{H} \boldsymbol{E}\right) .
\end{aligned}
$$

Define

$$
\begin{aligned}
& V=Y^{H} F \\
& U=E^{H} X .
\end{aligned}
$$

Hence

$$
\sum_{i=1}^{\varphi} \sigma_{i}\left(A B^{H}\right)=\Re \operatorname{tr}\left(\boldsymbol{\Lambda}_{a} \boldsymbol{V}^{H} \boldsymbol{\Lambda}_{b} U^{H}\right)
$$

Lsing Lemma A.1, the maximum of the right-hand side of (A.7) is given by the multiplication of the singular values of the diagonal matrices $\Lambda_{t}$ and $\boldsymbol{\Lambda}_{b}$. Thus, we have

$$
\sum_{i=1}^{4} \sigma_{i}\left(\boldsymbol{A} B^{H}\right) \leq \sum_{i=1}^{l} \sigma_{i}(\boldsymbol{A}) \sigma_{i}(\boldsymbol{B})
$$

and the proof is complete.

\section{APPENDIX B}

Minimization of the SlbSpace FitTing ERror

In this Appendix, we prove Theorem 1. The error of the two-sided unitary transformation is given by

$$
\begin{aligned}
\mathcal{E} & =\left\|A-U B V^{H}\right\|^{2} \\
& =\|A\|^{2}+\|B\|^{2}-2 \Re \operatorname{tr}\left(A V B^{H} U^{H}\right) .
\end{aligned}
$$

Minimization of (B.1) with respect to the choice of $U$ and $V$ is identical to maximization of

$$
\operatorname{Ulax}_{\mathrm{U}, \mathrm{V}} \Re \operatorname{tr}\left(\boldsymbol{A V B} \boldsymbol{B}^{H} \boldsymbol{U}^{H}\right)
$$

subject to $V$ and $U$ being unitary transformations. From Lemma A.l, it is seen that the maximum value of (B.2) is given by $\sum_{i=1}^{q} \sigma_{i}(\boldsymbol{A}) \sigma_{i}(\boldsymbol{B})$. Let us represent the singular value decomposition of the two matrices $A$ and $B$ by

$$
\begin{aligned}
& \boldsymbol{A}=\boldsymbol{E} \boldsymbol{\Lambda}_{a} \boldsymbol{F}^{H} . \\
& \boldsymbol{B}=\boldsymbol{X} \boldsymbol{\Lambda}_{t} \boldsymbol{Y}^{H} .
\end{aligned}
$$

Then, it is straightforward to see that with

$$
\begin{aligned}
& U=E X^{H} \\
& \boldsymbol{V}=F \boldsymbol{Y}^{H}
\end{aligned}
$$

the maximum is achieved. This completes the proof.

\section{REFERENCLS}

[1] M. Wax. T. Shan. and T. Kailath. "Spatio-temporal spectral analysis by eigenstructure methods." IEEE Trans. Acust. Speech, Signal Processing. vol. ASSP-32, pp. 817-827. Aug. 1984.

[2] H. Wang and M. Kaveh. "Coherent signal-subspace processing for the detection and estimation of angles of arrival of multiple wide-band sources,"IEEE Trans. Acumst, Speech. Signal Processing. vol. ASSP-33 pp. 823-831, Aug. 1985.

[3] R, O. Schmidt, "Mu!tiple emilter location and signal parameler estimation," IEEE Trans. Antemas Propagat., vol. 34. pp. 276-280, Mar 1986.

[4] S. Valaee and P. Kabal. "Selection of the focusing frequency in wideband array processing-MUSIC and ESPRIT," Proc, I6th Biemial Symp. Commun. (Kingston), 1992, pp. 410-414.

[5] K. M. Buckley and L. J. Griftiths, "Broad-band signal-subspace spatialspectrum (BASS-ALE) estimation," IEEE Trans. Acoust., Speech, Signal Processing. vol. 36, pp. 953-964, July 1988.

[6] J. Krolik and D. N. Swingler. "Multiple broad-band source location using stcered covariance malrices." IEEE Tians. Acus.st. Speech. Signal Prokessing. vol. 37, pp. 1481-1494, Oct. 1989.

[7] H. Hung and M. Kaveh. "Focusing matrices for coherent signal-subspace processing." IEEE Trans, Acoust. Speeth. Signat Protessing, vol. 36 pp. 1272-1281. Aug. 1988

[8] R. A. Horn and C. A. Johnson, Matris Analysis. Cambridge, England: Cambridge Lniversity Press, 1985.

19] C. Topics in Matrix Analysis. Cambridge, England: Cambridge Lniversity Press, 1991.

[!o] G. H. Golub and C. F. V. Loan, Matrix Computations, Baltimore. MD John Hopkins University Press. 1983.

II1] M. Wax, "Detection of coherent and noncoherent signals via the stochastic signal mudel," in Proc. IEEE Int Conf' Acoust., Speech, Signal Processing, May 1991. pp. 3541-3544.

[12] S. Validee and $P$. Kahal. "Detecion of the number of signals using prediclive slochastic complexily." in Pron'. IEEE Int. Conft. Acrust. Speedh, Signed Processing. Mar. 1992. pp. V-345-V-348.

I I.3] D. N. Swingler and J Krolik, "Source location bias in the coherently focused high-resolition broad-band beantormer." IEEE Trans. Acrust. Speedh. Signal Processing. vol. 37. pp. 14.3-145, Jan. 1989

14] T. W. Anderson. An Introdection to Multivariate Statistical Analssis. New York: Wiley, 1984.

[15] M. A. Doron and A. J. Weiss, "On focusing for wide-band array processing." IEEF Tians. Signal Processing. vol. 40. pp. 1295-1292. June 1992. 
[16] C. Zhou, F. Habre, and D. L. Jaggard, "A resolution measure for the MUSIC algorithm and its application to plane wave arrivals contaminated by coherent interference," IEEE Trans. Signal Processing, vol. 39 , pp. $454-463$, Feb. 1991

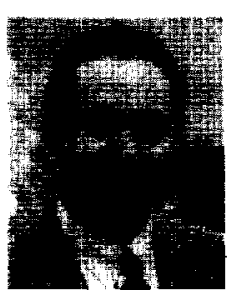

Shahrokh Valaee was born in Tabriz, lran, in 1961. $\mathrm{He}$ received the $\mathrm{B} . \mathrm{Sc}$. and $\mathrm{M} . \mathrm{Sc}$, degrees from Teheran University, Teheran. 1ran, and the Ph.D. degree from McGill University. Montreal, Canada. all in electrical engineering.

While at McGill, he was a research and senior teaching assistunt in communications and signal processing courses. He also has three years of experience in designing PABX systems. Presently, he is à research associate at IVRS-Télécommunications, Université du Québec, where he works on congestion control in B-ISDN. His research interests include detection and estimation, modeling and order selection. spectrum estination and harmonic retrieval, beamforming and array processing, and. most recently. networks.

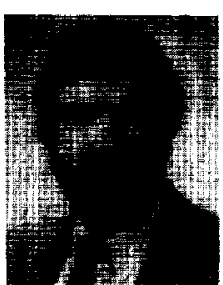

Peter Kabal (S70-M'75) received the B.A.Sc., M.A.Sc., and $\mathrm{Ph} . \mathrm{D}$. degrees in electrical engineering from the University of Toronto. Toronto. Canada. He is a Professor with the Department of Electrical Engineering at McGill University, Montreal, Cunada, and a Visiting Professor at INRSTélecommunications, which is a research instiute affiliated with the Université du Québec, Verdun. Canada. From September 1982 to September 1983, he was a full-time consultant to the Speech Communications Group at Bell-Northem Research, Verdun, Canada. From September 1987 to June 1988, he spent a sabbatical year as a Visiting Professor at the University of California at Santa Barbara. His current research interests focus on signal processing as applied to speech coding, adaptive filtering, and data transmission. 